\title{
Prosodic Phonological Representations Early in Visual Word Recognition
}

\author{
Jane Ashby \\ University of Massachusetts at Amherst
}

\author{
Andrea E. Martin \\ New York University
}

\begin{abstract}
Two experiments examined the nature of the phonological representations used during visual word recognition. We tested whether a minimality constraint (R. Frost, 1998) limits the complexity of early representations to a simple string of phonemes. Alternatively, readers might activate elaborated representations that include prosodic syllable information before lexical access. In a modified lexical decision task (Experiment 1), words were preceded by parafoveal previews that were congruent with a target's initial syllable as well as previews that contained 1 letter more or less than the initial syllable. Lexical decision times were faster in the syllable congruent conditions than in the incongruent conditions. In Experiment 2, we recorded brain electrical potentials (electroencephalograms) during single word reading in a masked priming paradigm. The event-related potential waveform elicited in the syllable congruent condition was more positive $250-350 \mathrm{~ms}$ posttarget compared with the waveform elicited in the syllable incongruent condition. In combination, these experiments demonstrate that readers process prosodic syllable information early in visual word recognition in English. They offer further evidence that skilled readers routinely activate elaborated, speechlike phonological representations during silent reading.
\end{abstract}

Keywords: phonology, syllables, reading, word recognition, minimality

Numerous word recognition studies have demonstrated that phonology plays a role in reading, yet the nature of readers' phonological representations is just beginning to be examined. An improved understanding of the nature of phonological representations in visual word recognition helps to conceptualize the interface between spoken language systems and reading-specific processes and, thus, holds implications for developing computational models of word recognition and eye movement control during reading. The present experiments investigated the nature of the phonological representations used in word recognition with two experimental paradigms: a modified lexical decision experiment and an event-related potentials (ERP) experiment. Specifically, we asked whether early phonological representations are complex enough to include suprasegmental, syllable information.

One central question about the nature of early phonological representations involves the claim of minimality or the idea that the phonological representations used in word recognition contain

Jane Ashby, Department of Psychology, University of Massachusetts at Amherst; Andrea E. Martin, Department of Psychology, New York University.

The research reported here partially fulfilled the requirements for Jane Ashby's doctoral dissertation at the University of Massachusetts at Amherst. The work was initially supported by a predoctoral Kirschstein National Research Service Award from the National Institutes of Health (HD045056) and was later supported by National Institutes of Health Grant HD051700. We thank the members of the dissertation committee, Gert DeVries, John Kingston, Alexander Pollatsek, Keith Rayner, and Lisa Sanders, for their suggestions, as well as Charles Clifton for his comments on the article. We appreciate the training in data analysis and interpretation received from Ina Bornkessel and Matthias Schlesewsky at the Max Planck Institute for Human Cognitive and Brain Science.

Correspondence concerning this article should be addressed to Jane Ashby, Department of Psychology, 661 Tobin Hall, University of Massachusetts, MA 01003. E-mail: ashby@psych.umass.edu only the minimal amount of information necessary to access a unique lexical entry (Frost, 1998). Frost's (1998) strong phonological theory of word recognition holds that phonological representations are necessarily activated at some point in word recognition, but early phonological representations contain only a sketch of the available phonological information. Lexical access activates a full phonological representation that is retrieved from memory. According to Frost (1998), a minimality constraint on early phonological representations permits speedy lexical access and accounts for the inconsistent phonological effects observed in previous lexical decision research (see Rastle \& Brysbaert, 2006, for a recent review).

One implication of the minimality constraint is a potential difference in the early activation of consonants and vowels. Frost (1998) proposed that early phonological representations might not include specific vowel information. For example, he claimed that the vowel phoneme in pint need not be fully resolved in order to access that particular lexical item. However, Ashby, Treiman, Kessler, and Rayner (2006) presented evidence that the early phonological representations used in silent reading do specify particular vowel phonemes. Ashby et al. (2006) utilized a boundary-change paradigm developed by Rayner (1975) in two parafoveal preview experiments to examine whether readers activated a specific vowel in an upcoming target word before the target is fixated. The vowel-same condition used previews (droon) in which the vowel phoneme was biased toward the vowel in the target word (drool), whereas the vowel-different condition used previews (drook) that were biased away from the phonemic vowel of the target word (drool). In both experiments, readers recognized target words faster in the vowel-same condition than when target words were preceded by previews with a different phonemic vowel. These data indicate that readers begin activating specific vowel information in advance of fixation and, thus, are inconsistent with 
Frost's proposal that early phonological representations contain unspecified vowel segments.

Of course, it is quite possible that early phonological representations include specified vowel segments but are still minimal compared with the phonological representations used in processing spoken language. Several linguistic theories (e.g., Bybee, 2001; Frauenfelder \& Lahiri, 1989; Selkirk, 1982) hold that spoken language representations contain multiple layers of sublexical information: a prosodic level that indicates the syllable structure, a skeletal level that codes the consonant (C) and vowel (V) pattern, and a melodic level that represents the contrasting properties of speech segments. Figure 1 depicts such a model, which is adapted from Clements and Keyser (1983). We refer to this model as an elaborated phonological representation. If the phonological representations in reading and speaking are similarly rich (Liberman, Shankweiler, \& Liberman, 1989), then skilled readers ought to activate phonological information at multiple levels. Alternatively, the phonological representations used in visual word recognition might be minimal in the sense that they contain all phoneme segments but omit suprasegmental, syllable information. For the purpose of this article, we claim that elaborated phonological representations contain suprasegmental as well as segmental information, whereas minimal representations contain segmental information only.

There are several reasons to doubt that the early phonological representations used in silent reading include suprasegmental information. Suprasegmental information (e.g., onset-rime structure, syllable boundaries, or lexical stress pattern) about a word's phonology is not explicitly encoded in English orthography. Therefore, the elaborated phonological representation described in Figure 1 may not be activated early in word recognition because it is not readily available in the initial visual stimulus. Also, if early phonological representations primarily function to support a visual lexical access route, then these supplemental representations might include only minimal content in order to maximize efficiency (Frost, 1998). Furthermore, a simplified, minimal representation that contained only phonological segments could account for the ubiquitous homophone effects that constitute the bulk of the evidence for phonological processing in word recognition.

However, naming and lexical decision experiments in several languages have demonstrated that readers activate elaborated phonological representations that include suprasegmental, syllable information. Carreiras, Alvarez, and de Vega (1993) initially reported a syllable frequency effect in visual word recognition in Spanish. Recently, Carreiras and Perea (2002) found that masked primes of the exact initial syllable facilitated lexical decision more than did primes with one more or one less letter. In an earlier experiment, Perea and Carreiras (1998) demonstrated syllable neighborhood effects on lexical decision and word naming times in Spanish. In French, Ferrand (2000; Ferrand \& New, 2003) dem-

Word:

Prosodic level (suprasegmental)

Skeletal level (segment)

Melodic level (features)

[k ae n

\section{CANDY}

$\begin{array}{lllll}\sigma & & & & \\ \mathrm{V} & \mathrm{C} & \mathrm{C} & \mathrm{V} \\ \mathrm{ae} & \mathrm{n} & & \mathrm{d} & \mathrm{i}]\end{array}$

Figure 1. Phonological representation in spoken language. onstrated that the number of syllables in words or nonwords affected naming and lexical decision latencies. In Chinese, Chen, Lin, and Ferrand (2003) found shorter naming latencies when targets were preceded by masked primes with the same initial syllable, even in the presence of mismatched tone. Hutzler, Conrad, and Jacobs (2005) conducted naming and lexical decision experiments in German that suggested that words with higher frequency first syllables took longer to recognize than did words with lower frequency first syllables. In sum, previous research suggests that readers of several languages activate syllable information in elaborated phonological representations.

However, the experimental evidence for the use of elaborated phonological representations by readers of English is currently inconclusive. At least one lexical decision experiment and several naming studies have failed to find evidence for the representation of suprasegmental, syllable information (Ferrand, Segui, \& Humphreys, 1997; Schiller, 1999, 2000). In contrast, eye movement studies of word recognition during the silent reading of sentences suggest that readers do activate elaborated phonological representations. Ashby and Rayner (2004) conducted a preview experiment to examine whether readers use parafoveally presented syllable information to facilitate word recognition. In their second experiment, first fixation durations were faster for target words (e.g., demand) preceded by a preview of the target's exact initial syllable (de) compared with previews containing one letter more than the exact initial syllable (dem). The syllable effect observed in this initial experiment was recently replicated with low-frequency words presented in sentence contexts (Ashby, 2006).

In summary, the evidence from naming experiments in English that is consistent with minimal phonological representations outweighs the evidence for elaborated representations at this time (Schiller, 1999, 2000). However, naming studies in several other languages have found evidence for elaborated early representations. To confuse matters more, two recent eye movement studies suggest that elaborated phonological representations are activated parafoveally when silently reading in English (Ashby, 2006; Ashby \& Rayner, 2004). These inconsistencies invite further investigation of whether skilled readers include suprasegmental information in the prelexical phonological representations used to recognize isolated words.

The present experiments used two different techniques to investigate the nature of the phonological representations used in visual word recognition. In Experiment 1, we measured fixation durations and reaction times in a modified lexical decision task. In Experiment 2, we recorded event-related potentials as participants read single words silently and made semantic decisions about filler items. In both experiments, participants read words (e.g., gender) preceded by two types of preview (or prime) information. In the syllable congruent conditions, the preview (gen) matched the initial syllable of the target exactly. In the syllable incongruent conditions, the preview ( $g e$ ) contained one letter more or less than the target's initial syllable. We predicted that syllable-congruent previews would facilitate word identification relative to syllableincongruent previews. The observation of a syllable congruency effect would suggest that readers processed suprasegmental information early in word recognition and, thus, activated elaborated phonological representations. 


\section{Experiment 1}

This experiment examined whether participants use elaborated phonological representations to retain parafoveally obtained syllable information during a saccade in a modified lexical decision task. An eye-contingent display change technique (Rayner, 1975, 1978) was used to present parafoveal previews that were syllabically incongruent and congruent with CV-initial (genocide) and CVC-initial (gender) target words. Figure 2 illustrates the boundary-change lexical decision paradigm (Henderson, Dixon, Petersen, Twilley, \& Ferreira, 1995; Rayner, McConkie, \& Zola, 1980), which offered readers advance parafoveal information about a word. Participants first fixated a cross on the left side of the computer screen, saw a row of $X$ s, then moved their eyes to the target on the right and decided whether it was a word. During the time they were programming the saccade to the target (150-250 $\mathrm{ms}$ ), advance information about the word was available parafoveally. When the eyes crossed an invisible boundary during the saccade, it triggered the computer to change the parafoveal preview to the target word.

The boundary-change lexical decision paradigm differs from a traditional lexical decision task in one critical way. In a traditional lexical decision task, participants view primes and identify words foveally and, therefore, are not required to retain prime information across a saccade. In contrast, the boundary-change lexical decision paradigm presents the preview parafoveally and requires participants to preserve the prime information over a saccade in order to facilitate word identification. Finding a syllable congruency effect in the present experiment would implicate the involvement of phonological memory processes to preserve information across a saccade.

Although this task is not a substitute for natural reading, it does minimize a few sources of variability that contribute noise to fixation time data collected during sentence reading. In sentence reading, variation in the launch site of saccades to the target affects the visibility of the preview and the foveal processing load on the previous fixation, and these factors affect the extent of parafoveal processing (Rayner, 1998). In contrast, previews in a boundarychange lexical decision task are presented at a constant visual angle (about $2^{\circ}$ ), and foveal vision is not occupied by word recognition processes.

In addition to investigating the parafoveal activation of elaborated phonological representations in isolated word recognition, we wanted to examine whether the syllable congruency effect reported by Ashby and Rayner (2004) was affected by differences in initial-bigram and initial-trigram frequencies between the two groups of target words. It is possible that initial string frequency contributed to the syllable effect, and this could suggest that the effect is at least partially orthographic in origin. To test this

\begin{tabular}{|c|c|c|}
\hline Fixation 1 & $\mathrm{xxx} \mid$ & se \\
\hline & & * \\
\hline Fixation 2 & $\mathrm{x} \times \mathrm{x} \mid$ & secret \\
\hline
\end{tabular}

Figure 2. A boundary-change lexical decision paradigm. A fixation point is denoted by the asterisk, and the vertical pipe indicates the invisible boundary. possibility, we chose the materials in the present experiment such that CV-and CVC-initial target words were matched on initial trigram.

The predictions for this experiment are straightforward. We expected lexical decision times (LDTs) to be faster for words preceded by syllable-congruent previews than for syllableincongruent previews in both the CV-and CVC-initial words. If the congruency effect is primarily phonological, it should appear even when materials are matched for initial trigram. This result would confirm the validity of the syllable congruency effect reported previously (Ashby, 2006; Ashby \& Rayner, 2004) and suggest that syllable information is part of the phonological representation that preserves preview information across a saccade. Replication of a syllable effect would necessarily indicate that early phonological representations contain prosodic phonological information. Alternatively, if initial-trigram frequency drove the apparent syllable effect in previous studies, then counterbalancing the previews in the present experiment should eliminate it. Therefore, a null result would suggest that orthographic processing of familiar letter sequences contributed to the preview effects observed in previous experiments.

\section{Method}

Participants. Thirty-two students at the University of Massachusetts at Amherst were paid \$7 or received experimental credit to participate in the experiment. All participants were native English speakers with normal vision who were naive about the purpose of the experiment.

Apparatus and procedure. The stimuli were presented on an NEC 4FG monitor through a VGA card that was controlled by a personal computer with a Pentium processor. An analog-to-digital converter interfaced the computer with a Generation-V dualPurkinje-image eye tracker. The monitor displayed text at a 200-Hz refresh rate that permitted display changes within $5 \mathrm{~ms}$. The eye tracker monitored movements of the right eye, although viewing was binocular. Letters were formed from a $7 \times 8$ array of pixels, using the fixed-pitch Borland $\mathrm{C}$ default font. Participants sat $61 \mathrm{~cm}$ away from a computer screen and silently read single words while their head position was stabilized by a bite bar. At this viewing distance, 3.8 letters occupied one degree of visual angle.

The eye-tracking system was first calibrated for the participant. At the beginning of each trial, a check calibration screen appeared, and participants who showed a discrepancy between where their eye fixated and the location of the calibration squares were recalibrated before the next trial. After the experimenter determined that the eye tracker was correctly calibrated, the participant was instructed to look at the calibration square on the far left of the screen and then the experimenter presented the trial (see Figure 2). At the start of each trial, participants fixated a cross $(+)$. The $X X X X$ appeared in foveal view, replacing the fixation cross, at the same time that a parafoveal preview appeared in the target region $2^{\circ}$ to the right. Participants were instructed to watch for the $X \mathrm{~s}$, then look at the target region, and decide whether it contained a word. While looking at the $X \mathrm{~s}$ and programming their next saccade (150-250 ms), participants could process the preview parafoveally, although they were never instructed to do so. Presentation of the target word was triggered during the saccade into the target region; as the eyes crossed an invisible boundary placed after the most rightward $X$, the target appeared (see Figure 2). The display 
change completed during the saccade, and participants rarely noticed any change. The participant clicked a response key to indicate a word (right) or a nonword (left) and proceeded to the next trial. Then the check calibration screen appeared before the next trial. The experiment was completed in one session of approximately 30 min.

Materials. Sixty-four target words (see Appendix A) were presented randomly interspersed with 84 unrelated words. The 148 words in the experiment were interspersed with 152 nonword trials. Target words consisted of two groups: CV initial (e.g., genocide) and CVC initial (e.g., gender). Because many words in English are ambisyllabic, we confirmed the syllabification of our materials with the MRC Psycholinguistic Database (Wilson, 1988) and Merriam-Webster's Collegiate Dictionary (1995). These sources appear to follow the traditional syllabification rule for CVCCV words of dividing between the two adjacent consonants (e.g., gen.der). Words were preceded by partial word previews that contained either the first two or the first three letters of the target, followed by underscores for the remainder of the word (e.g., ge___ and gen___ ( ) The CV-initial and CVC-initial words had a mean frequency of 19 occurrences per million words and 9 occurrences per million words, respectively (Francis \& Kučera, 1982). CV, CVC, and nonword foils had a mean length of 5.8, 6.7, and 6.9 letters, respectively. Foils were created by changing one or two of the final letters in polysyllabic real words (e.g., debane and yondes). Nonword foils were preceded by two or three letter primes (50\% of each). Filler words were preceded by whole word primes.

Design. Each participant read every target word once, with each target preceded by one of its two possible previews. Trials were fully counterbalanced, such that participants read half of the CV-initial and CVC-initial words preceded by congruent previews and half preceded by incongruent previews. Participants read each trigram-matched pair preceded either by a preview that was syllabically congruent in both cases (e.g., genocide preceded by ge___ and gender preceded by gen__) or incongruent in both cases (e.g., genocide preceded by gen___ and gender preceded by ge___ $)$.

\section{Results}

Lexical decision time (LDT) was the dependent variable. Total LDT measured the time between the start of the trial and the button press that ended the trial, which included saccade latency time. Mean saccade latency was $235 \mathrm{~ms}$ for the included trials. Foveal LDT measured the time spent looking at the word from the start of fixation until the keypress indicates a decision. Preview type (incongruent or congruent) was treated as a within factor in both the participant and item analyses. Word type (CV initial or CVC initial) was treated as a within factor in the participant analysis and a between factor in the item analysis. Participant counterbalancing group was included as a between factor in the analyses in order to remove variance due to list in both lexical decision measures (Pollatsek \& Well, 1995). Target word frequency was included as a covariate in the item analyses.

The data set was trimmed in the following way. Trials were excluded from data set if the total LDT was over $1,500 \mathrm{~ms}$, if the foveal LDT exceeded 1,200 ms, if the fixation time on the $X \mathrm{~s}$ was under $150 \mathrm{~ms}$, if the display change occurred before the eyes landed in the target region, or if the word was incorrectly judged to be a nonword. Following standard practice in display change experiments (Sereno \& Rayner, 1992), we set a data criterion at $75 \%$ to ensure that each participant contributed an adequate number of data points. The data from participants who met the $75 \%$ criterion for usable data (26 out of the original 32 participants) were submitted to analyses of variance (ANOVAs) by participants $\left(F_{1}\right)$ and items $\left(F_{2}\right)$. Four items were excluded from the data set, as they contributed fewer than five data points (from a possible 12 per condition), resulting in the means for $28 \mathrm{CV}$-initial words and 32 CVC-initial words being analyzed. As a result, four CVC words did not have a corresponding $\mathrm{CV}$ partner with the same trigram. ${ }^{1}$

Lexical decision accuracy. The mean accuracy of lexical decisions was $93 \%$. Syllable congruency did not affect accuracy rates $\left(F_{\mathrm{S}}<1\right)$. Participants were somewhat more accurate on $\mathrm{CV}$-initial targets $(95 \%)$ than on CVC-initial targets (92\%).

Total LDT. Table 1 contains the mean total LDTs for target words in the syllable-incongruent and syllable-congruent conditions. Reaction times were $18 \mathrm{~ms}$ faster on average when words were preceded by a syllable-congruent preview than a syllableincongruent preview, $F_{1}(1,24)=6.18, p<.05, F_{2}(1,55)=6.20$, $p<.05$, which indicates that syllable information in the preview was used in word identification. The interaction of word type and preview congruency was not significant $\left(F_{\mathrm{s}}<1\right)$, and this suggests that the number of segments in the preview did not affect total decision time.

Other trends in these data are reported here for completeness, although these are not relevant to our test of the minimality constraint. A main effect of word type (CV vs. CVC initial syllable) was significant in the participant analysis, $F_{1}(1,24)=$ $32.28, p<.001$, but not in the item analysis, $F_{2}(1,55)=2.35, p<$ .15. Mean reaction times may have been slower to the CVC-initial words because they were one letter longer on average and had a lower frequency of occurrence. Also, an interaction between counterbalancing condition and preview congruency was significant in the item analysis, $F_{2}(1,55)=41.58, p<.001$, but not in the participant analysis, $F_{1}(1,24)<1$. This trend in the item analysis appeared due to the faster LDTs contributed by one counterbalancing group of participants to half of the items.

Foveal LDT. Table 2 contains the mean foveal LDTs for target words in the syllable-incongruent and syllable-congruent conditions. Mean fixation times were $22 \mathrm{~ms}$ faster on average when words were preceded by syllable-congruent previews than by syllable-incongruent previews, $F_{1}(1,24)=13.41, p<.001, F_{2}(1$, $55)=3.84, p<.05$. This indicates that participants processed the syllable information in the preview and used it in identifying the word. The interaction of word type and preview congruency was not significant $\left(F_{\mathrm{S}}<1\right)$, and this suggests that the number of letters in the preview did not affect word identification time in this experiment.

Other trends in these data, which are not relevant to our test of the minimality constraint, are reported here. A main effect of word type (CV vs. CVC initial syllable) was significant in the participant analysis, $F_{1}(1,24)=19.20, p<.001$, but not in the item analysis

\footnotetext{
${ }^{1}$ The full set of CVC words remained in the analysis, as all contributed more than five data points per condition. Restricting the analysis to 28 items for both the CV and CVC words did not substantially change the pattern of mean reaction times; reaction times in the congruent condition were $19 \mathrm{~ms}$ shorter for $\mathrm{CV}$-initial words and $25 \mathrm{~ms}$ shorter for CVC-initial words compared with the incongruent condition.
} 
Table 1

Total Lexical Decision Time (ms) in Experiment 1

\begin{tabular}{lccc}
\hline \multicolumn{1}{c}{ Word } & $\begin{array}{c}\text { Incongruent } \\
\text { preview }\end{array}$ & $\begin{array}{c}\text { Congruent } \\
\text { preview }\end{array}$ & Difference \\
\hline CV initial & 979 & 966 & 13 \\
CVC initial & 1,024 & 1,001 & 23 \\
\hline
\end{tabular}

Note. $\mathrm{C}=$ consonant; $\mathrm{V}=$ vowel

$F_{2}(1,55)=1.61, p<.25$. Mean fixation times may have been longer to the CVC-initial words because they were longer and had a lower frequency of occurrence. An interaction between counterbalancing condition and preview congruency was significant in the item analysis, $F_{2}(1,55)=30.12, p<.001$, but not in the participant analysis, $F_{1}(1,24)=1.03, p<.50$.

Additional analyses. A review of the materials indicated that many CV-initial and CVC-initial pairs differed in the first syllables' vowel sound (e.g., secret/section). This pattern raises the possibility of a confound in the data. The observed differences in the incongruent and congruent conditions could stem from differences in the vowel sound activated by the preview (see Ashby et al., 2006; Henderson et al., 1995), rather than the syllable congruency of the preview. For example, it may be that a preview of sec activates the / $/$ / unit, which is the correct vowel sound in section but not in secret, and that this vowel match is the true source of the facilitation found in the congruent condition. To test this idea, we selected the five CV-initial and CVC-initial word pairs that had the most similar initial vowel phonemes: vesicle/vespers, genocide/ gender, solar/solstice, velocity/velvet, and delicious/deltoids. None of these pairs have the tense/lax vowel contrast found in secret/ section. For these five pairs of items, the vowel phoneme in the CVC preview had a similar relationship to the first vowel phoneme in both the CV- and CVC-initial target words. For example, the similarity of the vowels in vesicle and vespers means that the vowel phoneme in ves was similar to the initial vowel phoneme in each of those words. Discerning readers will notice that the relative similarity of the initial vowels in these five item pairs varies somewhat: The initial vowels in the first two pairs are the same, the vowels in the third pair are not quite the same, and the vowels in the last two pairs are still less similar (due to a stress shift that reduced the vowel in the $\mathrm{CV}$ member of each pair). Inspection of the mean foveal LDTs for these items (see Appendix B) does not suggest any systematic relationship between vowel similarity and the congruency effect.

We conducted a post hoc test of whether vowel activation was primarily responsible for our apparent congruency effect by comparing the mean LDTs in this subset of vowel-similar items with the LDTs for items with a tense/lax vowel contrast (secret/section). If vowel activation was the core of our preview effect, then that effect should be smaller in the subset of vowel-similar pairs than in the vowel-contrast pairs. This was not the case, however. For the vowel-similar items, the mean total LDTs were $33 \mathrm{~ms}$ faster to targets preceded by syllable-congruent previews than syllableincongruent previews compared with $17 \mathrm{~ms}$ for vowel-contrast items. Likewise, mean foveal LDTs were $43 \mathrm{~ms}$ faster in the congruent condition than in the incongruent condition compared with $14 \mathrm{~ms}$ for vowel-contrast items. As the effect size is numerically larger for the vowel-similar items than for the vowel- contrast items, the vowel contrast between the prime and target seems unlikely to be the source of the syllable congruency effect.

\section{Discussion}

In this experiment, we used a boundary-change lexical decision paradigm to present parafoveal preview information that could facilitate word identification if participants preserved that information across a saccade. The question was whether participants would preserve the preview information as a simple representation of phonemes (or possibly letters) or as an elaborated representation that included suprasegmental phonological information (i.e., syllables). The observation of faster LDTs to words preceded by syllable-congruent previews, compared with syllable-incongruent previews, suggests that readers represented syllable information available in the parafoveal previews and preserved that information across a saccade. Finding this result with trigram-matched materials indicates that initial-letter frequency was not responsible for the syllable effect. Thus, the syllable effect is unlikely to be orthographic in nature.

We recognize that some readers may disagree about the syllabification of the CVC-initial words with pronounceable medial consonant clusters (e.g., basket). This disagreement could reflect a tendency toward ambisyllabicity in a minority of our materials (9 out of 64 words). However, Schiller (2000) found no effect of letter number or syllable congruency on naming latencies for the ambisyllabic targets presented in his Experiment 5. Thus, we expect that any ambisyllabicity in our targets would work against the demonstration of a syllable effect.

Experiment 1 provides converging evidence for suprasegmental levels of phonological activation in a lexical decision task that complements the parafoveal syllable effect observed during sentence reading (Ashby, 2006; Ashby \& Rayner, 2004). Because this lexical decision experiment is the first to demonstrate initialsyllable priming effects in English, it is tempting to conclude that early phonological representations preserve syllable information over the course of the saccade to the parafoveal word.

It is possible that similarity between the vowel phoneme in the preview and in the target word contributes to the syllable congruency effect, although the present experiment does not provide positive evidence for this finding (see Appendix B). Any such contribution raises an interesting question: How would a particular vowel phoneme be preferentially activated by a preview? Orthographic vowels are ambiguous in English, as any vowel letter can be produced in a lax, tense, or reduced manner. It seems likely that the syllable information in the preview string could guide the relative activation of potential vowel phonemes (e.g., CVC syllables could preferentially activate lax vowels). This is a question for future experiments, but claiming a role for phonological vowels in word recognition, as established in Ashby et al.'s (2006) study

Table 2

Foveal Lexical Decision Time (ms) in Experiment 1

\begin{tabular}{lccc}
\hline \multicolumn{1}{c}{ Word } & $\begin{array}{c}\text { Incongruent } \\
\text { preview }\end{array}$ & $\begin{array}{c}\text { Congruent } \\
\text { preview }\end{array}$ & Difference \\
\hline CV initial & 699 & 675 & 24 \\
CVC initial & 730 & 709 & 21 \\
\hline
\end{tabular}

Note. $\quad \mathrm{C}=$ consonant $\mathrm{V}=$ vowel. 
under somewhat different circumstances, does not preclude a syllable congruency effect.

Previous reading research indicates that skilled readers activate prosodic information when silently reading sentences (Ashby, 2006; Ashby \& Clifton, 2005; Ashby \& Rayner, 2004). Ashby and Rayner (2004) found a syllable effect in their parafoveal preview experiment, yet a syllable effect did not appear when the prime was presented foveally in a fast-priming paradigm. Ashby and Rayner attributed the different results in the two experiments to the different memory demands imposed by each display-change technique. In the preview experiment, parafoveal syllable information had to be preserved across the saccade to the target in order to facilitate word recognition, whereas the unmasked foveal prime information did not need to be retained in memory. The idea that the representation of suprasegmental phonological information arises from preserving the preview (or prime) information in memory is tested more directly in Experiment 2.

\section{Experiment 2}

This experiment examined the time course of activation of suprasegmental information and the role of memory processes in phonological representation. It is possible that readers only activate elaborated phonological representations when preview information appears parafoveally, as in sentence reading. Alternatively, such representations might be activated whenever advance information must be stored in memory, leading to the prediction that masked foveal priming will also yield a syllable effect in word recognition. Experiment 2 recorded electrical potentials at the scalp while participants silently read single words (pilot) preceded by two or three letter primes that were congruent or incongruent with the initial syllable of the target word. Figure 3 describes the four-field masked priming paradigm used in this experiment. Because a mask following the prime obscures the visual stimulus, readers can use the prime information to facilitate word recognition if they preserve it in memory. If readers process initial syllable structure en route to word recognition, then the ERP waveforms elicited in the syllable-congruent condition should differ significantly from waveforms elicited in the syllable-incongruent condition.

The time course of phonological representation has been investigated in several ERP studies, yet the nature of these representations is largely unknown. Early studies typically used discrimination-type tasks, in which participants made rhyme judgments about pairs of words. These rhyme judgment experiments often reported phonological effects between 300 and $500 \mathrm{~ms}$ posttarget (Rugg, 1984; Rugg \& Barrett, 1987). More recent stud-

$\begin{array}{lc}\text { Start (100 ms) } & + \\ \text { Forward Mask (98 ms) } & \text { \#\#\#\# } \\ \text { Prime (42 ms) } & \text { PIL\#\# or PI\#\#\# } \\ \text { Backward Mask (98 ms) } & \text { pilot } \\ \text { Target Word (644 ms) } & \end{array}$

Figure 3. A four-field masked priming paradigm. ies have measured phonological processing in semantic decision experiments, in which participants read target words and pseudowords embedded in highly predictable sentence contexts at the terminal position (e.g., Newman \& Connolly, 2004). The targets varied with respect to their semantic and phonological appropriateness for the sentence context, and the phonological effect appeared as a reduced negativity around $400 \mathrm{~ms}$ for pseudowords that were homophonous with the expected target, relative to the negativity produced by a semantically inappropriate word or nonword that sounded different from the expected target. Although these experiments are informative about phonological processing in highly predictive sentence contexts, their contribution to understanding prelexical phonological processing in the course of normal reading is uncertain. It is possible that the phonological effects observed around $400 \mathrm{~ms}$ may be more relevant to phonological decision making or sentence interpretation than online phonological processing during word recognition $(\mathrm{Ku}-$ tas \& Hillyard, 1984; Kutas \& Van Petten, 1990).

A few ERP studies have found phonological effects earlier than 300 ms posttarget. Kramer and Donchin (1987) recorded electroencephalogram (EEG) as participants read sequentially presented word pairs and decided whether they rhymed. The words pairs were orthographically similar and rhymed, orthographically similar and did not rhyme, orthographically different and rhymed, and orthographically different and did not rhyme. Waveform differences at $260 \mathrm{~ms}$ posttarget indicated that participants detected rhyme in orthographically different words fairly early in word recognition. Niznikiewicz and Squires (1996) examined the relative time course of phonological and semantic processing during word recognition by recording EEG as participants read words in sentence contexts and made sentence acceptability judgments. Target words appeared in four conditions: a correct sentence completion, a homophonous and orthographically similar word, an orthographically similar word that was nonhomophonous, or an unrelated word. They reported an early effect around $200 \mathrm{~ms}$ in the form of an increased negativity to incorrect homophone completions relative to correct completions. Niznikiewicz and Squires interpreted this effect as an N200 component, or a mismatch negativity between the given phonological form and its conflicting orthographic forms, which indicates the use of phonological information in silent reading. This interpretation of the N200 appears consistent with data reported by Pexman, Lupker, and Reggin (2002) that suggest that homophone effects in lexical decisions are initially triggered by phonological processing. However, the Pexman et al. (2002) study also indicated that homophone effects in lexical decision actually arise from competition in the orthographic system (i.e., the feedback inconsistency effect reported in Stone, Vanhoy, \& Van Orden, 1997). Thus, the N200 effect may indicate the early operation of phonological and orthographic processes, rather than primarily a phonological effect.

Several ERP experiments have investigated phonological processing in languages other than English. Bentin, MouchetantRostaing, Giard, Echallier, and Pernier (1999) examined the ERP correlates of orthographic, phonetic, phonological, and semantic processing in French. Participants read words, pseudowords, and nonwords in four variations of an oddball paradigm while their EEG was recorded. Waveform differences between 270 and 320 $\mathrm{ms}$ poststimulus indicate that participants distinguished between pronounceable and nonpronounceable targets, irrespective of lexical status. This phonological/phonetic effect involved a greater 
negativity to pronounceable targets than did nonpronounceable targets. In contrast, maximum semantic effects appeared in waveform differences at $450 \mathrm{~ms}$ posttarget. In a similar study, Simon, Bernard, Largy, Lalonde, and Rebai (2004) examined orthographic and phonological effects on early ERP components as participants read words, nonwords, and symbol strings silently. Their results indicated that the N230 and N320 components were sensitive to phonology as well as lexicality and orthography.

Two recent ERP experiments examined syllable frequency effects in word recognition. Barber, Vergara, and Carreiras (2004) recorded ERPs as participants made lexical decisions to high- and low-frequency Spanish words with high- and low-frequency initial syllables. They observed an increased positivity to low-frequency syllables compared with high-frequency syllables at $200 \mathrm{~ms}$ posttarget, whereas word frequency did not elicit differences in amplitude until 300-400 ms posttarget. Hutzler et al. (2004) examined syllable frequency effects in German and found an increased positivity to words with low-frequency first syllables between 190 $\mathrm{ms}$ and $280 \mathrm{~ms}$ compared with words with high-frequency first syllables. The later appearance of a lexicality effect suggests a prelexical locus for this syllable frequency effect.

In a recent study, Carreiras, Vergara, and Barber (2005) examined the effect of syllable congruency on word recognition in Spanish. Participants made lexical decisions to CV-initial and CVC-initial words presented in two colors. In half of the target trials, the color boundary coincided with the syllable boundary, and in the other half, the boundaries did not coincide. Carreiras et al. (2005) recorded EEGs as participants made lexical decisions to words, nonwords, and pseudowords. Whereas they did not find any effect of coinciding color and syllable boundaries on reaction times, inconsistency between the syllable boundary and the color boundary did modulate the ERP waveforms between 180 and 260 $\mathrm{ms}$, such that potentials evoked in the incongruent condition were more positive than potentials evoked in the congruent and single color conditions. This difference in brain potentials had an anterior scalp distribution and was replicated in their second experiment. In contrast, the lexicality effects observed around $400 \mathrm{~ms}$ were distributed more centrally on the scalp, such that the incongruent condition elicited less negativity than the single color condition.

In summary, the previous research indicates that phonological effects can appear substantially earlier than lexical effects in the ERP record, arising between $180 \mathrm{~ms}$ and $320 \mathrm{~ms}$ posttarget (Barber et al., 2004; Bentin et al., 1999; Carreiras et al., 2005; Hutzler et al., 2004; Simon et al., 2004). We expected a syllable congruency effect to appear in that time window.

\section{Method}

Participants. Twenty students at the University of Massachusetts at Amherst and Hampshire College were paid $\$ 12$ or received experimental credit to participate in the experiment. All participants were native English speakers with normal vision.

Apparatus and procedure. Electrical potentials were detected at the scalp via a 32-channel electrode cap with silver/silverchloride electrodes arranged in the 10-20 international system (Jasper, 1958) and digitized with a Neuroscan amplifier. A mastoid electrode served as the reference electrode during recording. The EEG was recorded at a sampling rate of $500 \mathrm{~Hz}$ while participants read single words preceded by masked primes on a Macintosh black and white monitor set to a display rate of $75 \mathrm{~Hz}$. Superlab software (http://www.superlab.com) presented the materials and recorded manual responses. The EEG was rereferenced to averaged mastoids offline. ERPs were created by epoching around triggers placed at the onset of words of interest, baseline corrected to $200 \mathrm{~ms}$ prestimulus onset, and artifact rejected over horizontal and vertical eye-electrodes for deflections greater than $70 \mu \mathrm{V}$. Then the EEG for each electrode was averaged across trials for each participant in each condition, yielding individual averages that were included in the grand average for each condition. Data from an additional 4 participants were excluded from the grand averages due to technical difficulties.

Participants were instructed to read each word silently as it appeared on the screen. Trials appeared in black letters on a white background and were viewed through a 3 -in. $\times 5$-in $(7.62 \mathrm{~cm} \times$ $12.7 \mathrm{~cm}$ ) window cut into a large piece of white cardboard designed to mask the surrounding visual field. At the start of each trial, a fixation cross appeared at the center of the screen (see Figure 3). A forward mask appeared, then a partial-word prime followed by a backward mask, and then the target word. Semantic judgments were made on half of the filler trials via keypress Questions (e.g., "Is it clothing?") were presented after the target disappeared from the screen. The experiment was completed in one session of approximately $90 \mathrm{~min}$.

Materials. Participants read $50 \mathrm{CV}$-initial words (pilot) and 50 CVC-initial words (magnet; see Appendix C). Unlike the previous experiment, here the $\mathrm{CV}$-initial and $\mathrm{CVC}$-initial words did not generally share the same orthography. However, they were comparable in length and frequency. CV-initial words ranged from 5 to 8 letters in length and CVC-initial words were 6 to 9 letters in length (overall mean $=6.3$ letters). Both groups of target words had a mean frequency of two occurrences per million (Francis \& Kučera, 1982). As in Experiment 1, the syllabification patterns of CV-initial and CVC-initial targets were confirmed by two sources (Wilson, 1988; Merriam-Webster, 1995). Filler items were similar in length, frequency, and morphology to the experimental items.

Design. Each participant read the 100 target words interspersed with 198 unrelated filler items that were also preceded by masked primes. ${ }^{2} \mathrm{CV}$-initial and CVC-initial target words were presented randomly. Each participant read every target word once, preceded by one of its two possible primes. A three-letter prime served as the syllable-incongruent condition for the CV-initial words, whereas a two-letter prime served as the syllable-congruent condition. The inverse was true for the CVC-initial words. Material lists and participant groups were fully counterbalanced.

Data analysis. Twenty-five electrodes ( 5 rows $\times 5$ columns) were selected a priori for the analyses. This group excluded the two most frontal sites (FP1 and FP2) as well as the posterior three electrodes (O1, OZ, and $\mathrm{O} 2)$. The missing values for two noisy electrodes (TP7 and T7) were interpolated for 3 participants by taking the mean value of the three nearest neighbors. All the effects reported here were also significant at the 15 electrode sites that required no interpolation (i.e., the middle three columns: F3, FZ, F4, FC3, FCZ, FC4, C3, CZ, C4, CP3, CPZ, CP4, P3, PZ, and

\footnotetext{
2 The planned design included 50 words in each of the two groups. After data collection, we reviewed the script file and found four coding errors. Two CV-initial items were miscoded as fillers and two CV-initial items were miscoded as CVC-initial items. These errors reduced the number of $\mathrm{CV}$-initial items and introduced some unnecessary noise into the data.
} 
P4). Figure 4 presents the waveforms for the horizontal and vertical eye electrodes in the congruent and incongruent conditions and indicates the absence of serious artifacts in the time window of interest (250-350 ms).

Waveform differences that occurred earlier than $100 \mathrm{~ms}$ posttarget were attributed to low-level visual effects and, thus, were not of interest. Inspection of the present waveforms focused our analyses between $250 \mathrm{~ms}$ and $350 \mathrm{~ms}$ posttarget onset. Initial ANOVAs treated target type (CV initial or CVC initial), syllable congruency of prime (incongruent or congruent), and electrode position ( 5 rows $x 5$ columns) as within-participant factors in a $2 \times$ $2 \times 5 \times 5$ analysis of the time window from $250 \mathrm{~ms}$ to $350 \mathrm{~ms}$. There was no main effect of target type (CV initial or CVC initial; $F<1)$ nor any Target Type $\times$ Syllable Congruency interaction $(F<1){ }^{3}$ Therefore, the following ANOVAs tested the significance of the syllable congruency effect.

\section{Results}

Figure 5 displays the waveforms elicited by the congruent and incongruent conditions at three midline electrodes. The waveform elicited in the syllable-congruent condition appears more positive between $250 \mathrm{~ms}$ and $350 \mathrm{~ms}$ compared with the waveform for the syllable-incongruent condition, $F(1,19)=7.629, p<.01$, and congruency did not interact with any factors (all $F \mathrm{~s}<1$ ). To determine the onset of the syllable congruency effect, we analyzed two time windows separately (225-250 ms and $250-275 \mathrm{~ms}$ ). In terms of the onset of the congruency effect, we tested significance conservatively at the midline 15 electrode sites ( 2 [number of letters] $\times 2$ [syllable congruency] $\times 3$ [columns] $\times 5$ [rows]), avoiding any interpolation. The syllable congruency effect was not significant between $225 \mathrm{~ms}$ and $250 \mathrm{~ms}, F(1,19)=1.625, p<.25$, but was significant between $250 \mathrm{~ms}$ and $275 \mathrm{~ms}, F(1,19)=4.864$, $p<.05$, and this confirms the onset of the effect by $250 \mathrm{~ms}$.

\section{Discussion}

Experiment 2 investigated whether suprasegmental syllable information presented briefly in masked primes modulated the ERPs
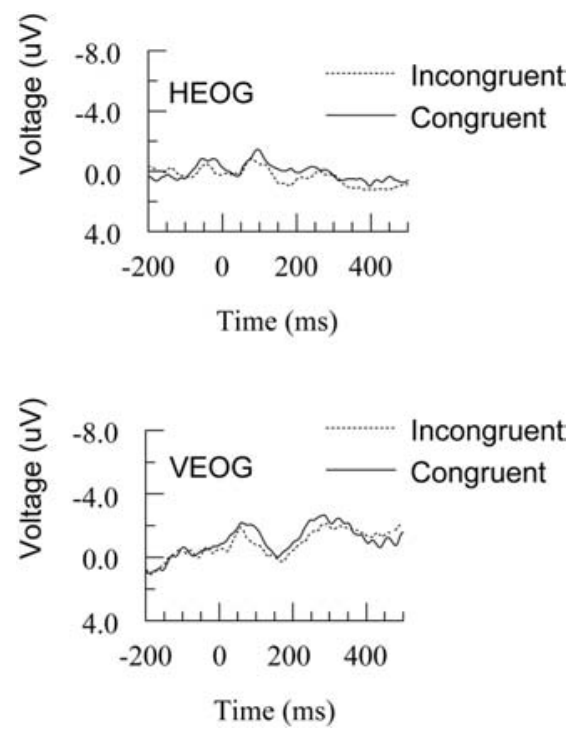

Figure 4. Averaged electrical potentials at the horizontal (HEOG) and vertical (VEOG) eye electrodes.
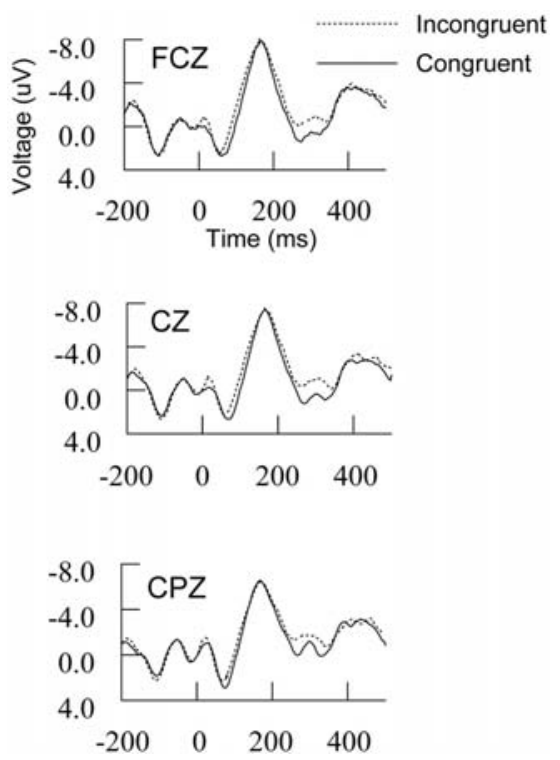

Figure 5. Averaged electrical potentials at midline frontal-central, central, and central-parietal electrode.

elicited during passive word reading. Between $250 \mathrm{~ms}$ and $350 \mathrm{~ms}$, ERPs were more positive to targets preceded by syllable-congruent primes than were syllable-incongruent primes. This syllable effect reached significance within the time window that Hutzler et al. (2004) found their syllable frequency effect (190-280 ms), and its duration overlaps with the 270-320 ms window in which Bentin et al. (1999) and Simon et al. (2004) found phonological effects. Identifying a syllable congruency effect in the ERP record suggests that readers activated elaborated phonological representations during visual word recognition.

In general, this experiment extends the syllable congruency effect observed by Carreiras et al. (2005) to masked priming in English. However, the data from these two studies differ in several ways. First, the present syllable effect appeared somewhat later in our experiment (250-350 ms) than in Carreiras et al.'s (2005) study (180-260 ms). Second, our effect had a broad scalp distribution, whereas the Carreiras et al. studies found an anterior distribution. Third, we found an increased negativity when words were preceded by syllable incongruent primes, whereas Carreiras et al. observed a decreased negativity in the incongruent condition. Given that the Carreiras et al. studies manipulated the congruency of color and syllable boundaries, whereas the present study used two- and three-letter primes, we are not surprised by these apparent differences. Differences in effect onset, scalp distribution, and polarity could result from each task eliciting activity from different

\footnotetext{
${ }^{3}$ Word type (CV or CVC initial) did interact with the syllable congruency of the prime between $125 \mathrm{~ms}$ and $250 \mathrm{~ms}$ posttarget. This interaction indicated a difference between potentials elicited in the two-letter prime condition and the three-letter prime condition, $F(1,19)=9.422, p<.01$. Because this difference could be attributed either to the number of letters in the prime or to the degree of orthographic overlap between prime and target, we are unable to draw any firm conclusions about the origin of the effect. No syllable congruency effect appeared in this time window $(F<$ 1). Likewise, no word type interactions appeared 250-350 ms posttarget $(F<1)$.
} 
neuron populations. Recent research by Holcomb and Grainger (2006) suggests that task effects may be responsible. They used a masked priming paradigm similar to the one in the present study and found that less related whole word primes elicited a broadly distributed increased negativity compared with identity primes at 250, 325, and $400 \mathrm{~ms}$. Thus, it seems that onset and polarity of our syllable congruency effect (increased negativity in the incongruent condition) may be the more typical result in a masked priming paradigm.

These data extend the parafoveal syllable effect observed in Experiment 1 and in previous studies to foveal masked priming. The present syllable effect indicates that neither a parafoveal presentation of the prime nor an intervening saccade is necessary to obtain a syllable effect. However, this result does suggest that some interval between the prime and the target (e.g., a backward mask) is sufficient to obtain a syllable congruency effect. A comparison between the present data and the lack of a syllable effect reported by Ashby and Rayner (2004) in a fast-priming experiment (discussed previously) also supports the suggestion that some interruption of the visual stimulus between prime and target is necessary for a syllable effect to arise. This is consistent with previous eye movement research (Ashby \& Rayner, 2004).

In summary, the syllable effect observed in this study indicates that readers use elaborated phonological representations in visual word recognition and implicates a role for memory processes in activating suprasegmental information. Because elaborated representations are also activated when processing spoken language, the present result could indicate that skilled readers initiate access to spoken language representations en route to lexical access. Additionally, detecting a syllable effect in this experiment demonstrates that a partial-word masked-priming paradigm can be combined with ERP data collection methods to yield a sensitive technique for investigating the nature of the phonological representations used in word recognition.

\section{General Discussion}

The main theoretical question posed in this article was whether readers use minimal representations for lexical access, as suggested by the strong phonological theory of word recognition (Frost, 1998). Two experiments tested whether readers activate elaborated phonological representations that include suprasegmental information early in visual word recognition. If a minimality constraint operates on early phonological representations, then advance prosodic information should not affect word reading. However, the data collected in two different experimental paradigms demonstrate a consistent effect of initial-syllable congruency in word recognition.

In Experiment 1, reaction times were recorded during a boundary-change lexical decision task to test whether a syllable congruency effect would appear in lexical decision when syllable information appeared parafoveally. We observed faster LDTs to targets preceded by syllable congruent previews compared with previews with one more or one less letter. This result suggests that readers activated phonological information beyond the segment level before fixating the target word. In Experiment 2, we recorded EEG as participants silently read words preceded by syllablecongruent and syllable-incongruent masked primes. Waveform differences indicative of word processing appeared in the ERP record between 250 and $350 \mathrm{~ms}$. In this time window, the syllable- congruent condition elicited an increased positivity, relative to the syllable-incongruent condition. Thus, the present experiments offer converging evidence that skilled readers typically activate elaborated phonological representations in visual word recognition.

Because both experiments provided initial syllable information in advance of the target, rather than requiring readers to parse it out of a lexical prime, the following caveat applies. The elaborated phonological representations activated during natural reading might typically include syllable information, or they may simply have the capacity to include syllable information. In either case, the present results are inconsistent with the minimality constraint proposed in the strong theory of phonology in word recognition (Frost, 1998). Generally speaking, processing suprasegmental information in advance of fixation should not be possible if readers only activated minimal representations of phonemic segments. In this respect, our claims differ from those of Carreiras et al. (1993), in that we do not anticipate that readers of English decompose words into syllables during word recognition.

Because any challenge to the minimality constraint hinges on evidence that readers initiate elaborated phonological representations early in word recognition and en route to lexical access, it might be helpful to explain why we regard the source of the syllable effect as primarily prelexical. In the present experiments, the previews and masked primes consisted of partial-word fragments. Because the target words appeared in random order and without any sentence context, lexical access was not possible at the time the syllable information was presented. In order for syllable information to affect word recognition, participants had to activate the suprasegmental information in the prime before a lexical entry was available. Furthermore, when we used targets matched for initial trigram (e.g., gender/genocide) in Experiment 1, we controlled for effects of cohort activation that could possibly arise. Because participants activated prosodic representations before lexical access was possible, the source of the syllable effect is prelexical by definition. In contrast, the time window in which differences between the incongruent and congruent waveforms appeared in the ERP record (250-350 ms) probably reflects processes that made use of the syllable information obtained from earlier elaborated phonological representations. Although we acknowledge that early phonological representations may be impoverished relative to the full representations activated at lexical access, it is difficult to reconcile the present data with the claim that readers activate only the minimal information needed to access a unique lexical entry (Frost, 1998).

In addition to challenging the validity of the minimality constraint, the present experiments make two novel contributions to the word recognition research. First, these experiments found a syllable effect in visual word recognition in English. This suggests that readers of English activate phonological representations that are similar to those activated by readers in other languages (e.g., German, Spanish, and French). Second, it is interesting that the syllable effect appeared in isolated word recognition. Four previous eye movement studies reported prosodic processing during the silent reading of sentences (Ashby, 2006; Ashby \& Clifton, 2005; Ashby \& Rayner, 2004; Hirotani, Frazier, \& Rayner, 2006). In each study, it was speculated that readers begin activating sentence-level prosody to support later comprehension processes (Fodor, 1998). In the present experiments, it seems unlikely that sentence-level prosody motivated the activation of elaborated rep- 
resentations (given the lack of sentence context). Rather, these experiments suggest a different locus for the syllable effect. It is possible that the prosodic structure of early phonological representations emerges when advance information about a word is stored in memory, either during a saccade to a word (as in Experiment 1) or during a backward mask (as in Experiment 2). Although further research is needed to confirm a link between memory and elaborated phonological representations in reading, these data suggest the viability of such a link. If suprasegmental representations arise in the course of memory activity, then the activation of elaborated phonological representations might be automatic in natural silent reading in which skilled readers routinely begin processing words parafoveally. It is possible that word recognition experiments that do not supply advance information about target words effectively eliminate the context in which phonology begins to operate and, thus, may underestimate the role of phonology in natural reading. Alternatively, elaborated phonological representations may be detectable only when there is sufficient time between preview and target for them to emerge. Future experiments may explore these possibilities.

Finding that readers activate phonological representations in word recognition might be unremarkable in a writing system that encodes suprasegmental information. However, as syllable information is not encoded explicitly in written English, evidence for the activation of elaborated phonological representations in visual word recognition is of interest. If syllable information is not encoded in the text, and if it cannot distinguish between lexical items, then why do readers include suprasegmental information in their phonological representations? We suspect that elaborated, prosodic phonological representations are activated automatically in the process of preserving advance parafoveal information across the saccade to the target. It is possible that such advance prosodic information contributes to the activation of complete phonological representations that influence when and where the eyes move during reading (Ashby \& Clifton, 2005).

Readers' capacity to activate complex phonological representations early in word recognition challenges researchers' current understanding of the role of phonology in reading and invites speculation about the point at which reading processes interface with spoken language. To the extent that the elaborated phonological representations used in word recognition resemble spoken language representations, the syllable effect reported here suggests that the reading system might interface with spoken language processes early in word recognition through the word's phonological representation. Skilled readers' elaborated phonological representations are speechlike in the sense that they can include suprasegmental, prosodic information that is not encoded in the writing system. Obviously, it is possible that early reading representations differ from spoken language representations in some way, and future experiments may demonstrate what those differences are. Currently, the similar nature of phonological representations in word recognition and spoken language representations points to the intriguing possibility that skilled readers begin activating spoken language representations in the early phases of visual word recognition.

\section{References}

Ashby, J. (2006). Prosody in skilled silent reading: Evidence from eye movements. Journal of Research in Reading, 29, 318-333.
Ashby, J., \& Clifton, C., Jr. (2005). The prosodic property of lexical stress affects eye movements during silent reading. Cognition, 96, B89-B100.

Ashby, J., \& Rayner, K. (2004). Representing syllable information during silent reading: Evidence from eye movements. Language and Cognitive Processes, 19, 391-426.

Ashby, J., Treiman, R., Kessler, B., \& Rayner, K. (2006). Vowel processing during silent reading: Evidence from eye movements. Journal of Experimental Psychology: Learning, Memory, and Cognition, 32, 416424.

Barber, H., Vergara, M., \& Carreiras, M. (2004). Syllable frequency effects in visual word recognition: Evidence from ERPs. NeuroReport, 15, $545-548$.

Bentin, S., Mouchetant-Rostaing, Y., Giard, M. H., Echallier, J. F., \& Pernier, J. (1999). ERP manifestations of processing printed words at different psycholinguistic levels. Journal of Cognitive Neuroscience, 11, $35-60$.

Bybee, J. (2001). Phonology and language use. Cambridge, England: Cambridge University Press.

Carreiras, M., Alvarez, C. J., \& de Vega, M. (1993). Syllable frequency and visual word recognition in Spanish. Journal of Memory and Language, 32, 766-780.

Carreiras, M., \& Perea, M. (2002). Masked priming effects with syllabic neighbors in a lexical decision task. Journal of Experimental Psychology: Human Perception and Performance, 28, 1228-1242.

Carreiras, M., Vergara, M., \& Barber, H. (2005). Early event-related potential effects of syllabic processing during visual word recognition. Journal of Cognitive Neuroscience, 17, 1803-1817.

Chen, J. Y., Lin, W. C., \& Ferrand, L. (2003). Masked priming of the syllable in Mandarin Chinese speech production. Chinese Journal of Psychology, 45, 107-120.

Clements, G. N., \& Keyser, S. J. (1983). CV phonology. Cambridge, MA: MIT Press.

Ferrand, L. (2000). Reading aloud polysyllabic words and nonwords: The syllabic length effect reexamined. Psychonomic Bulletin \& Review, 7 , 142-148.

Ferrand, L., \& New, B. (2003). Syllabic length effects in visual word recognition and naming. Acta Psychologia, 113, 167-183.

Ferrand, L., Segui, J., \& Humphreys, G. W. (1997). The syllable's role in word naming. Memory \& Cognition, 25, 458-470.

Fodor, J. D. (1998). Learning to parse? Journal of Psycholinguistic Research, 27, 285-319.

Francis, W. N., \& Kučera, H. (1982). Frequency analysis of English usage. Boston: Houghton-Mifflin.

Frauenfelder, U. H., \& Lahiri, A. (1989). Understanding words and word recognition: Can phonology help? In I. W. M.-W. (Ed.), Lexical representation and process (pp. 319-341). Cambridge, MA: MIT Press.

Frost, R. (1998). Toward a strong phonological theory of visual word recognition: True issues and false trails. Psychological Bulletin, 123, 71-99.

Henderson, J. M., Dixon, P., Petersen, A., Twilley, L. C., \& Ferreira, F. (1995). Evidence for use of phonological representations during transsaccadic word recognition. Journal of Experimental Psychology: Human Perception and Performance, 21, 82-97.

Hirotani, M., Frazier, L., \& Rayner, K. (2006). Punctuation and intonation effects on clause and sentence wrap-up: Evidence from eye movements. Journal of Memory and Language, 54, 425-443.

Holcomb, P. J., \& Grainger, J. (2006). On the time course of visual word recognition: An event-related potential investigation using masked repetition priming. Journal of Cognitive Neuroscience, 18, 1631-1643.

Hutzler, F., Bergmann, J., Conrad, M., Kronbichler, M., Stenneken, P., \& Jacobs, A. M. (2004). Inhibitory effects of first syllable-frequency in lexical decision: An event-related potential study. Neuroscience Letters, 372, 179-184.

Hutzler, F., Conrad, M., \& Jacobs, A. M. (2005). Effects of syllable 
frequency in lexical decision and naming: An eye movement study. Brain and Language, 92, 138-152.

Jasper, H. (1958). The ten-twenty electrode system of the International Federation. Electroencephalography and Clinical Neurophysiology, 10, 371-375.

Kramer, A. F., \& Donchin, E. (1987). Brain potentials as indices of orthographic and phonological interaction during word matching. Journal of Experimental Psychology: Learning, Memory, and Cognition, 13, $76-86$.

Kutas, M., \& Hillyard, S. A. (1984, January 12-18). Brain potentials during reading reflect word expectancy and semantic association. $\mathrm{Na}$ ture, 307, 161-163.

Kutas, M., \& Van Petten, C. (1990). Electrophysiological perspectives on comprehending written language. New Trends and AdvancesTechniques in Clinical Neurophysiology, 41, 155-167.

Liberman, I. Y., Shankweiler, D., \& Liberman, A. M. (1989). The alphabetic principle and learning to read. In D. Shankweiler \& I. Y. Liberman (Eds.), Phonology and reading disability: Solving the reading puzzle (pp. 2-33). Ann Arbor: University of Michigan Press.

Merriam-Webster's collegiate dictionary (10th ed.). (1995). Springfield, MA: Merriam-Webster.

Newman, R. L., \& Connolly, J. F. (2004). Determining the role of phonology in silent reading using event-related brain potentials. Cognitive Brain Research, 21, 94-105.

Niznikiewicz, M., \& Squires, N. K. (1996). Phonological processing and the role of strategy in silent reading: Behavioral and electrophysiological evidence. Brain and Language, 52, 342-364.

Perea, M., \& Carreiras, M. (1998). Effects of syllable frequency and syllable neighborhood frequency in visual word recognition. Journal of Experimental Psychology: Human Perception and Performance, 24, $134-144$.

Pexman, P. M., Lupker, S. J., \& Reggin, L. D. (2002). Phonological effects in visual word recognition: Investigating the impact of feedback activation. Journal of Experimental Psychology: Learning, Memory, and Cognition, 28, 572-584.

Pollatsek, A., \& Well, A. D. (1995). On the use of counterbalanced designs in cognitive research: A suggestion for a better and more powerful analysis. Journal of Experimental Psychology: Learning, Memory, and Cognition, 21, 785-794.

Rastle, K., \& Brysbaert, M. (2006). Masked phonological priming effects in English: Are they real? Do they matter? Cognition, 53, 97-145.

Rayner, K. (1975). The perceptual span and peripheral cues in reading. Cognitive Psychology, 7, 65-81.

Rayner, K. (1978). Foveal and parafoveal codes in reading. In J. Requin (Ed.), Attention and performance VII (pp. 149-162). Hillsdale, NJ: Erlbaum.

Rayner, K. (1998). Eye movements in reading and information processing: 20 years of research. Psychological Bulletin, 124, 372-422.

Rayner, K., McConkie, G. W., \& Zola, D. (1980). Integrating information across eye movements. Cognitive Psychology, 12, 206-226.

Rugg, M. D. (1984). Event-related potentials in phonological matching tasks. Brain and Language, 23, 225-240.

Rugg, M. D., \& Barrett, S. (1987). Event-related potentials and the interaction between orthographic and phonological information in a rhyme judgment task. Brain and Language, 32, 336-361.

Schiller, N. O. (1999). Masked syllable priming of English nouns. Brain and Language, 68, 300-305.

Schiller, N. O. (2000). Single word production in English. Journal of Experimental Psychology: Learning, Memory, and Cognition, 26, 512528.

Selkirk, L. (1982). The syntax of words. Cambridge: MIT Press.

Sereno, S. C., \& Rayner, K. (1992). Fast priming during eye fixations in reading. Journal of Experimental Psychology: Human Perception and Performance, 18, 173-184.

Simon, G., Bernard, C., Largy, P., Lalonde, R., \& Rebai, M. (2004). Chronometry of visual word recognition during passive and lexical decision tasks: An ERP investigation. International Journal of Neuroscience, 114, 1401-1432.

Stone, G. O., Vanhoy, M. D., \& Van Orden, G. C. (1997). Perception is a two-way street: Feedforward and feedback phonology. Journal of Memory and Language, 36, 337-359.

Wilson, M. D. (1988). The MRC psycholinguistic database: Machine readable dictionary, Version 2. Behavioural Research Methods, Instruments and Computers, 20, 6-11. 
Appendix A

Materials for Experiment 1

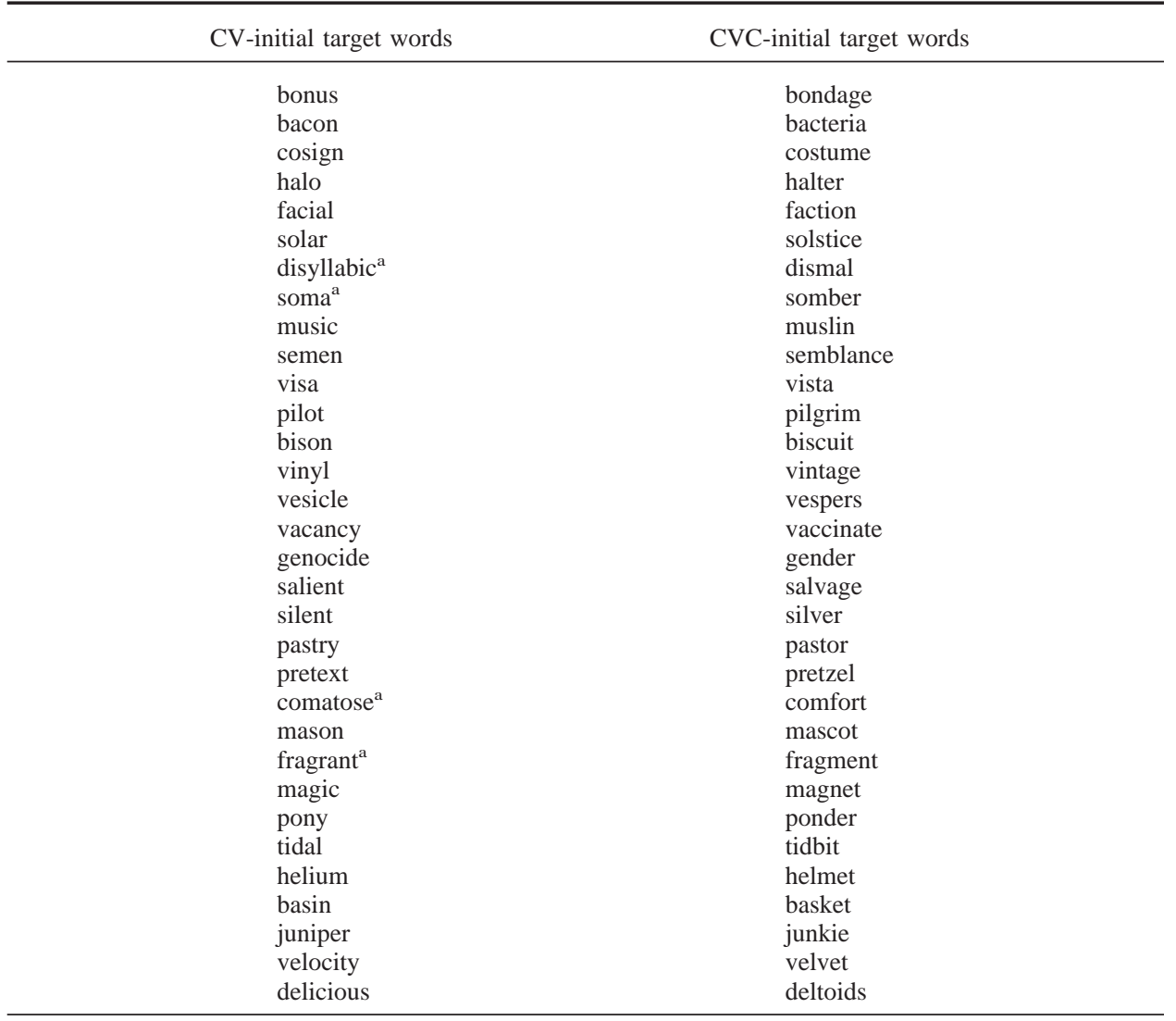

Note. $\mathrm{C}=$ consonant; $\mathrm{V}=$ vowel

atems with less than five data points were excluded from the statistical analyses.

Appendix B

Foveal Lexical Decision Time (ms) for Vowel-Similar Item Pairs in Experiment 1

\begin{tabular}{lccr}
\hline Word & Incongruent preview & Congruent preview & Difference \\
\hline Genocide & 742 & 719 & 23 \\
Gender & 676 & 598 & 78 \\
Vesicle & 824 & 718 & 106 \\
Vespers & 810 & 922 & -112 \\
Solar & 699 & 675 & 24 \\
Solstice & 861 & 716 & 145 \\
Velocity & 701 & 651 & 50 \\
Velvet & 727 & 640 & 87 \\
Delicious & 773 & 695 & 78 \\
Deltoids & 949 & 946 & 3 \\
\hline
\end{tabular}

(Appendixes continue) 


\section{Appendix C}

Materials for Experiment 2

\begin{tabular}{|c|c|c|c|}
\hline \multicolumn{2}{|c|}{ CV-initial target words } & \multicolumn{2}{|c|}{ CVC-initial target words } \\
\hline basin & nitrate & badminton & membrane \\
\hline botany & nudist & bondage & mildew \\
\hline bovine & pagan & cadmium & mundane \\
\hline cadence & pastry & camper & muslin \\
\hline cider & poker & censor & nectar \\
\hline cipher & possum & cinder & nostril \\
\hline covert & rabies & cistern & pelvis \\
\hline cushion & radiant & comrade & pompous \\
\hline decency & recipe & cosmos & rafter \\
\hline demon & reflex & culprit & rupture \\
\hline devious & rover & deltoid & rustic \\
\hline divers & salient & dismal & segment \\
\hline facial & savage & faction & semblance \\
\hline famine & savior & falter & sensual \\
\hline favors & sequel & fender & tactile \\
\hline feline & spider & festive & tamper \\
\hline fragrant & super & fumble & tempest \\
\hline futile & tigress & gambit & tidbit \\
\hline jovial & tulip & gender & velvet \\
\hline kosher & vacancy & halter & vintage \\
\hline latent & vacate & jasper & viscous \\
\hline migrant & vagrant & jumble & vulture \\
\hline minus & vibrant & lactate & wander \\
\hline modify & viper & lender & yonder \\
\hline motive & votive & magnet & zombie \\
\hline
\end{tabular}

Note. $\mathrm{C}=$ consonant $; \mathrm{V}=$ vowel.

Received June 5, 2006

Revision received November 28, 2006

Accepted March 2, 2007 